\title{
Predictive value of CONUT score combined withserum CA199 levels in postoperative survival of patients with pancreatic ductaladenocarcinoma: a retrospective study
}

\author{
Ankang Wang ${ }^{\text {Equal first author, } 1}$, Bo Sun ${ }^{\text {Equal first author, } 1}$, Min Wang ${ }^{2}$, Hao Shi $^{1}$, Zhiwei Huang $^{1}$, Tao He $^{1}$, Qiu Li $^{1}$, Jiaqi Deng $^{3}$ \\ , Wenguang Fu ${ }^{\text {Corresp., } 1}$, Yu Jiang ${ }^{\text {Corresp. } 1}$ \\ ${ }^{1}$ Department of Hepatobiliary Surgery, The Affiliated Hospital of Southwest Medical University, luzhou, china \\ 2 Department of Nutrition, The Affiliated Hospital of Southwest Medical University, luzhou, china \\ 3 Department of Ultrasound, The Affiliated Hospital of Southwest Medical University, luzhou, china \\ Corresponding Authors: Wenguang Fu, Yu Jiang \\ Email address: fuwg@swmu.edu.cn, 415943914@qq.com
}

Background: The preoperative controlling nutritional status (CONUT) score and serum carbohydrate antigen 199 (CA199) levels are individually correlated with the prognosis of pancreatic ductal adenocarcinoma (PDAC). The objective of this study aimed to investigate the efficacy of CONUT score and CA199 (CONUT-CA199) combination in predicting the prognosis of PDAC patients undergoing radical surgery.

Methods: We retrospectively analyzed the preoperative CONUT scores and serum CA199 levels of 294 patients with PDAC who underwent radical resection at the Affiliated Hospital of Southwest Medical University between March 2012 and July 2019. Patients were divided into four groups on the basis of their preoperative CONUT scores and serum CA199 levels: CONUT ${ }^{\text {low }} /$ CA199 $^{\text {low }}(1)$, CONUT $^{\text {low }} /$ CA199 $^{\text {high }}(2)$, CONUT $^{\text {high }} /$ CA199 $^{\text {low }}(3)$, and CONUT $T^{\text {high }} / C A 199^{\text {high }}$ (4). The prognostic effects were compared among the groups.

Results: CONUT ${ }^{\text {high }}$ was more frequent in patients with positive peripancreatic infiltration and ClavienDindo classification of $\geq 11$ la $(P<0.001)$. Kaplan-Meier analysis revealed obvious difference in overall survival (OS) and recurrence-free survival (RFS) among patients with PDAC having CONUT-CA199 scores of $1,2,3$ and $4(P<0.001)$. Peripancreatic infiltration, lymph node metastasis, pTNM stage, CONUT score, serum CA199 levels, and CONUT-CA199 classification were found to be the independent prognostic factors for OS and RFS in multivariate analyses. In time-dependent receiver operating characteristic (ROC) analyses, the area of the CONUT-CA199 score under the ROC curve (AUC) was higher than that of the preoperative CONUT score or serum CA199 levels for the prediction of OS and RFS.

Conclusion: CONUT-CA199 classification may be more effective in predicting the postoperative prognosis of PDAC patients 
1 Predictive value of CONUT score combined with serum CA199 levels in postoperative survival of patients with pancreatic ductal adenocarcinoma: a retrospective study

4 Ankang Wang ${ }^{1, \#}$, Bo Sun ${ }^{1, \#}$, Min Wang ${ }^{2}$, Hao Shi ${ }^{1}$, Zhiwei Huang ${ }^{1}$, Tao He ${ }^{1}$, Qiu 5 $\mathrm{Li}^{1}$, Jiaqi Deng ${ }^{3}$, Wenguang $\mathrm{Fu}^{1 *}, \mathrm{Yu} \mathrm{Jiang}^{1 *}$

6 'Department of Hepatobiliary Surgery, The Affiliated Hospital of Southwest

7 Medical University, Luzhou, China

$8{ }^{2}$ Department of Nutrition, The Affiliated Hospital of Southwest Medical University, 9 Luzhou, China

$10{ }^{3}$ Department of Ultrasound, The Affiliated Hospital of Southwest Medical

11 University, Luzhou,China

12 the email addresses for all authors:

13 Ankang Wang: wang2955@outlook.com

14 Bo Sun: 1363003969@qq.com

15 Min Wang: 12110875@qq.com

16 Hao Shi: 494217348@qq.com

17 Zhiwei Huang: hzwyy701401@163.com

18 Tao He: 411936526@qq.com 
19 Qiu Li: 354145933@qq.com

20 Jiaqi Deng: jiaq87@163.com

21 Wenguang Fu: fuwg@swmu.edu.cn

22 Yu Jiang: 415943914@qq.com

$23 *$ Corresponding author:

24 Wenguang $\mathrm{Fu}$

25 Department of Hepatobiliary Surgery, The Affiliated Hospital of Southwest

26 Medical University, Luzhou 646000, Sichuan Province, China.

27 E-mail: fuwg@swmu.edu.cn

28 Telephone: $+86-830-3165903$

$29 \quad$ Yu Jiang

30 Department of Hepatobiliary Surgery, The Affiliated Hospital of Southwest

31 Medical University, Luzhou 646000, Sichuan Province, China.

32 E-mail: 415943914@qq.com

33 Telephone: $+86-830-3165903$

34 \# Ankang Wang and Bo Sun contributed equally to this work and should be 35 considered as co-first authors. 
Abstract

Background: The preoperative controlling nutritional status (CONUT) score and serum carbohydrate antigen 199 (CA199) levels are individually correlated with the prognosis of efficacy of CONUT score and CA199 (CONUT-CA199) combination in predicting the prognosis of PDAC patients undergoing radical surgery.

Methods: We retrospectively analyzed the preoperative CONUT scores and serum CA199 level of 294 patients with PDAC who underwent radical resection at the Affiliated Hospital of Southwest Medical University between March 2012 and July 2019. Patients were divided into four groups on the basis of their preoperative CONUT scores and serum CA199 levels: CONUT ${ }^{\text {low/CA199low }}$ (1), CONUT low/CA199high $\quad$ (2), CONUThigh/CA199low (3), and CONUT ${ }^{\text {high/CA199high }}$ (4). The prognostic effects were compared among the groups.

Results: CONUT ${ }^{\text {high }}$ was more frequent in patients with positive peripancreatic infiltration and Clavien-Dindo classification of $\geq \mathrm{IIIa}(P<0.001)$. Kaplan-Meier analysis revealed obvious difference in overall survival (OS) and recurrence-free survival (RFS) among patients with PDAC having CONUT-CA199 scores of $1,2,3$ and $4(P<0.001)$. Peripancreatic infiltration, lymph node metastasis, pTNM stage, CONUT score, serum CA199 levels, and CONUT-CA199 classification were found to be the independent prognostic factors for OS and RFS in multivariate analyses. In time-dependent receiver operating characteristic (ROC) analyses, the area of the CONUT-CA199 score under the ROC curve (AUC) was higher than that of the preoperative CONUT score or serum CA199 levels for the prediction of OS and RFS.

Conclusion: CONUT-CA199 classification may be more effective in predicting the postoperative prognosis of PDAC patients.

Keywords: pancreatic ductal adenocarcinoma; controlling nutritional status score; 
carbohydrate antigen 199; prognosis

Pancreatic cancer (PC) is a malignant disease with strong invasiveness in humans, and it is expected to be the second leading cause of cancer related death in the future (Rahib et al. 2014). Pancreatic ductal adenocarcinoma (PDAC), which originates from the ductal epithelium, is the most common histological type of PC, accounting for approximately $95 \%$ of pancreatic exocrine tumors (Kamisawa et al. 2016). Currently, the only available treatment modality for PDAC is surgical resection (Gong et al. 2013). However, the disease is often diagnosed at a later stage owing to its initially unpredictable biological behavior. At that time, liver, lymph nodes, peripheral vessels, and nerves are often affected, with the tumor showing rapid growth, resulting in poor prognosis, and the 5-year survival rate has been stagnant at $6 \%$ for decades (El-Khayat et al. 2018; Siegel et al. 2019). Numerous studies have shown that tumor size, lymph node metastasis, vascular invasion and serum tumor markers (TMs) are vital prognostic factors for PC (Karamitopoulou et al. 2013; Staal et al. 2019; Winter et al. 2013), Moreover, early detection of postoperative recurrence can help improve the survival rate of patients with PDAC(Wu et al. 2019), therefore, it is important to determine the factors affecting the prognosis of these patients after pancreatectomy. It is not difficult to obtain the serum TMs level of patients from clinical data, which is of potential value for diagnosis, monitoring of postoperative recurrence and predicting survival rate (Fujioka et al. 2007). The serum marker CA199 has shown diagnostic potential in patients with latent and early PDAC (Haab et al. 2015; O'Brien et al. 2015), and can predict disease progression (Duffy et al. 2010; Satake et al. 1985).

Relevant reports have shown that the prognosis of tumor is closely related to the inflammatory status, immune function and nutritional status of patients(Mantzorou et al. 2017; Ni et al. 2019; van Dijk \& Pot 2016; Xiao et al. 2019). Numerous studies have found that malnutrition significantly increases postoperative complications and has a negative impact on the quality of life, hospital stay, anti-cancer treatment effect and overall survival in cancer patients(Borre et al. 2018; Fujiya et al. 2018; Lin et al. 2019).Furthermore, the latest research shows that there is a close relationship between nutritional status and prognosis of patients with cancer, including PDAC (Abe et al. 2018; Balzano et al. 2017; Gilliland et al. 2017). The 
90

91

92

controlling nutritional status (CONUT) score, a system for scoring immune nutritional status that emerged in 2005, has garnered the attention of researchers (Ignacio de Ulibarri et al. 2005), it includes the measurement of serum albumin and total cholesterol levels as well as peripheral blood lymphocytes. This scoring system has been considered as a predictor of prognosis for postoperative liver cancer, gastric cancer, colorectal cancer, and PDAC (Iseki et al. 2015; Kato et al. 2018; Shoji et al. 2017; Takagi et al. 2017). The patient's serum TMs are mostly determined by the tumor itself, whereas the CONUT score reflects the overall immune and nutritional status of patients. Both indicators demonstrate their role in assessing the prognosis of patients with PDAC. However, the value of their joint application is still unclear, this study aims to use these two indicators in combination to evaluate the prognosis of patients with PDAC.

\section{Materials and Methods}

\section{Study population}

All patients with $\mathrm{PC}$ who received radical resection in the affiliated hospital of the Southwest Medical University between March 2012 and July 2019 were retrospectively analyzed; a total of 294 cases met the inclusion criteria of this study. Inclusion criteria were as follows: patients 1) with histopathological confirmation of PDAC; 2) who had undergone radical resection; 3) who did not receive any neoadjuvant chemotherapy and/or radiotherapy before surgery; 4) with no history of other malignant tumors; 5) with complete clinical and follow-up data; and 6) in whom no metastatic lesions were found in the whole body before surgery. Exclusion criteria were as follows: patients 1) with acute or chronic infectious diseases preoperatively; 2) with preoperative complications of blood system diseases, kidney diseases, or cardiovascular and cerebrovascular diseases; 3) with any other known autoimmune disease; 4) with history of steroid medication use within 15 days before operation; 5) who received preoperative immune enhancement therapy or had a recent history of blood transfusion; and 6) who died within 30 days after operation.

\section{Investigational Variables}


All preoperative clinicopathological data were obtained from the electronic medical record system; the data included age, gender, height, weight, serum CA199 levels and carcinoembryonic antigen (CEA), tumor location, tumor size, histopathological type. Invasion and metastasis of peripancreatic, lymph nodes, lymphatic vessels and blood vessels, and pTNM staging were performed. Prognostic nutrition index (PNI) and CONUT score were calculated by blood routine results. Complications were presented by clavien-dindo classification and incidence of pancreatic fistula. Blood samples were collected 1 week before surgery and assessed for serum albumin and total cholesterol levels as well as total peripheral lymphocyte count. CONUT scores and PNI (Ignacio de Ulibarri et al. 2005; Pinato et al. 2012) were calculated according to previously described methods, as shown in Table 1. Postoperative complications were presented by the Clavien-Dindo classification system(Clavien et al. 2009) and the 2016 version of the postoperative pancreatic fistula grading system released by the International Study Group on Pancreatic Surgery (Bassi et al. 2017). The largest diameter of the tumor in the pathological sampling was considered as tumor size, and tumor staging was performed according to the TNM staging criteria of AJCC version 8 (van Roessel et al. 2018). We communicated with the patients before surgery and their consent was orally obtained for our study. Our research was supported by the Ethics Committee of the Affiliated Hospital of Southwest Medical University (NO.KY2019053).

\section{Follow-up}

We follow up all patients in a standardized way. Follow-up examination included abdominal ultrasound, chest X-ray, routine blood work, blood biochemistry (liver function, renal function), and TMs assessment. Contrast-enhanced computed tomography, magnetic resonance imaging, positron emission tomography, and other modalities were used depending on the situation if a suspicious lesion was detected and the nature of the lesion could not be defined. In accordance with the Chinese comprehensive guidelines for the diagnosis and treatment of PC (Pancreatic Cancer Committee of Chinese Anti-Cancer 2018), the patients were reexamined every 3 months during the first year after surgery, followed by every 3-6 months in next 2-3 
143

144

145

146

147

148

149

150

151

152

153

154

155

156

157

158

159

160

161

162

163

164

165

166

167

168

years. After 3 years, the follow-up period changed to 6 months. Survival data were obtained through patient outpatient visits and telephone follow-up. We counted the interval between the completion of surgery and death or the last follow-up, and the interval between the completion of surgery and tumor recurrence or the last follow-up, respectively expressed as the overall survival (OS) and Recurrence-free survival (RFS). Tumor recurrence included local recurrence and distant metastasis (liver and peritoneum, lungs, bone, etc.). The follow-up deadline was August 2019.

\section{Definition of preoperative CONUT-CA199 score}

The optimal cut-off value of preoperative CONUT score was 3, which was used as the criterion to divide 294 patients into low group $(<3 ; n=194)$ and high group $(\geq 3 ; n=100)$. Patients were divided into the following two groups according to the optimal cut-off value of serum CA199 levels (36.6 ng/mL): CA199low $(<36.6 ; \mathrm{n}=148)$, CA199high $(\geq 36.6 ; \mathrm{n}=146)$. Based on the cut-off values of preoperative CONUT and CA199, we defined the CONUTCA199 score. patients with CONUT ${ }^{\text {low }} /$ CA199low $(n=95)$ were assigned a score of 1 ; those with CONUT $^{\text {low }} /$ CA199high $(n=99)$ were assigned a score of 2 ; those with CONUThigh/CA199 ${ }^{\text {low }}(\mathrm{n}=$ 53) were assigned a score of 3 ; and those with CONUT ${ }^{\text {high/CA199high }}(\mathrm{n}=47)$ were assigned a score of 4 .

\section{Statistical analyses}

The classified data were summarized using a number (\%), and the difference between each group of variables is detected by chi-square test. A post hoc power analysis was completed. The power of the Peripancreatic infiltration and the Clavien-Dindo classification group was 0.64 and 0.98, respectively. The optimal cut-off values of CONUT score, CA199, CEA, age, size, PNI, and the area under the curve (AUC) were obtained by receiver operating characteristic (ROC) curve analysis. Survival curves were presented using the Kaplan-Meier method and the differences were compared by log-rank test. Firstly, univariate analysis was carried out for various clinical and pathological variables, and covariates with $P$ value $<0.05$ were included in 
multivariate analysis. Cox proportional hazard model and stepwise analysis were used to obtain independent influencing factors of OS and RFS. IBM SPSS Statistics package v.24.0 (Chicago, IL, USA) was used for statistical analysis, $P<0.05$ was considered statistically significant.

\section{Results}

294 patients who met the criteria were enrolled [163 men (55.4\%) and 131 women (44.6\%); age range, $29-78$ years; mean age, $55.5 \pm 10.8$ years].

Among the enrolled patients, $214(72.8 \%)$ had tumors in the pancreatic head, $63(21.4 \%)$ had tumors in the pancreatic body and tail, and $17(5.8 \%)$ had tumors that were diffuse in the pancreas. Among all patients, 131 (44.5\%) had poorly differentiated, 96 (32.7\%) had moderately differentiated, and 67 (22.8\%) had highly differentiated tumors. There were 70 (23.8\%), 125 (42.5\%), and $99(33.7 \%)$ patients with stage I, II, and III tumors, respectively. The general situation of the two groups of patients is shown in table 2. CONUThigh was more frequent in patients with positive peripancreatic infiltration and Clavien-Dindo classification $\geq$ IIIa $(P<$ 0.001).

The 5-year OS of the CONUT ${ }^{\text {low }}$ group $(11.0 \%)$ was significantly higher than that of the CONUT ${ }^{\text {high }}$ group $(2.9 \%)(P<0.0001)$ (Fig. 1A). The 5-year OS rate of the CA199high group (4.7\%) was lower than that of the CA199 ${ }^{\text {low }}$ group (13.3\%) $(P<0.013)$ (Fig. 1B).

Patients were divided into four groups to determine the impact of combining the CONUT scores and serum CA199 levels (CONUT-CA199) on prognosis. The 5-year OS rates of patients with CONUT ${ }^{\text {low/CA199low }}$ CONUT ${ }^{\text {low/CA199high }}$, CONUT ${ }^{\text {high} / C A 199 l o w, ~ a n d ~}$ CONUT $^{\text {high }} / \mathrm{CA} 199^{\text {high }}$ were $15.3 \%, 9.1 \%, 6.1 \%$, and $0 \%$, respectively $(P<0.0001)$ (Fig. 2A). In addition, the similar 5-year RFS rates were $9.2 \%, 7.9 \%, 4.7 \%$, and $0 \%$, respectively $(P<0.0001)$ (Fig. 2B).ROC analysis was used to further evaluate the effect of three independent factors on prognosis in our research. The results showed that the preoperative CONUT-CA199 scores were more predictive of OS and RFS in patients with PDAC than preoperative CONUT scores or 
194

195

196

197

198

199

200

201

202

203

204

205

206

207

208

209

210

211

212

213

214

215

216

217

218

219

preoperative serum CA199 levels alone (OS: $\mathrm{AUC}=0.685,95 \%$ CI: $0.625-0.746 ; P<0.001$; RFS: $\mathrm{AUC}=0.692,95 \%$ CI: $0.632-0.751 ; P<0.001 ;$ Fig. 3A-B)

Univariate analyses showed that age ( $<52$ vs. $\geq 52$ years; $P<0.05$ ), serum CA199 levels ( $<36.6$ vs. $\geq 36.6 \mathrm{ng} / \mathrm{mL} ; P<0.001)$, tumor size ( $<3.1$ vs. $\geq 3.1 \mathrm{~cm} ; P<0.05)$, histopathological type (poorly differentiated vs. moderate-highly differentiated; $P<0.001$ ), peripancreatic infiltration (positive vs. negative; $P<0.001$ ), lymph node metastasis (positive vs. negative; $P<$ 0.001 ), superior mesenteric artery invasion (positive vs. negative; $P<0.001$ ), portal vein system invasion (positive vs. negative; $\mathrm{P}<0.05$ ), nerve plexus invasion (positive vs. negative; $P<0.05$ ), pTNM stage (I/II vs. III; $P<0.001)$, PNI ( $<46.1$ vs. $\geq 46.1 ; P<0.001)$, the CONUT score (low vs. high; $P<0.001$ ), the CONUT-CA199 score ( 1 vs. 2 vs. 3 vs. $4 ; P<0.001)$ were related to OS and RFS (Table 3).

Since the CONUT-CA199 score includes the CONUT score and serum CA199 levels, two multi-factor Cox proportional models were set up to avoid colinearity problems. Among them, peripancreatic infiltration $(P<0.05)$, lymph node metastasis $(P<0.001)$, pTNM stage $(P<0.05)$, the CONUT score $(P<0.001)$, serum CA199 levels $(P<0.001)$, and the CONUT-CA199 score $(P<0.001)$ were independent prognostic factors for OS and RFS in multivariate analyses (Table 4).

\section{Discussion}

The CONUT score has been suggested as an indicator of immune-nutritional status of the host (Ignacio de Ulibarri et al. 2005; Tokunaga et al. 2017). Increasing body of documents have suggested that patients with high preoperative CONUT scores generally have poor nutritional and pro-tumor immunity status, potentially leading to tumor invasion and metastasis. A growing number of studies have shown that patients with high preoperative CONUT score are generally poorer in nutritional status and pro-tumour immunity status, and promote tumor invasion and metastasis (Liang et al. 2017; Shoji et al. 2017), which is significant for survival prognosis in postoperative patients with multiple cancers (Harimoto et al. 2018; Liu et al. 2018; Yang et al. 
220

221

222

223

224

225

226

227

228

229

230

231

232

233

234

235

236

237

238

239

240

241

242

243

244

245

246

2019a). Related studies have shown that the CONUT score is associated with survival prognosis of patients with unresectable PDAC and is an independent predictor of survival of patients with PDAC after pancreatectomy (Asama et al. 2018; Kato et al. 2018). Similar to previous reports, the finding of our report indicate that the preoperative CONUT score has value in predicting the postoperative prognosis of PADC patients. As the highest protein in human plasma produced by the liver, albumin can be used to assess the nutritional status of the body. Patients with low serum albumin levels are associated with poor nutritional and immune status, which can be a favorable condition for tumor invasion and metastasis (Liu et al. 2016). Lymphocyte expression in tumor defense is critical by inducing cytotoxic cell death. Therefore, a decrease in the amount of such cells in the blood may be related to impaired tumor immune function, allowing for tumor progression (Berntsson et al. 2016; Gooden et al. 2011; Jacobson 2006; Tang et al. 2014). PNI, which includes serum albumin levels and total lymphocyte count, is one of the most commonly used indicators of nutritional status (Kanda et al. 2011). It is known to be closely related to the prognosis of various cancers (Mohri et al. 2013; Sun et al. 2015; Yamamoto et al. 2019). Compared with PNI, the CONUT score includes the measurement of total serum cholesterol levels as well. Cholesterol, as an important component of cell membranes, is involved in many signaling pathways related to tumor development, progression, and immunogenicity; furthermore, cholesterol levels act as an important nutritional index (Haghikia \& Landmesser 2018; Jacobs et al. 2012; Yang et al. 2019b). Therefore, the CONUT score is considered to be a better nutritional and immune prognostic factor than PNI. In our study, PNI was found to be associated with OS and RFS of patients with PDAC after surgery, but it was not an independent predictor; however, the preoperative CONUT score was an independent predictor of OS and RFS in patients with PDAC after surgery.

The Clavien-Dindo grading system is currently the most commonly used statistical classification system for complications. High CONUT scores correlate with an increased incidence of postoperative pneumonia, length of hospital stay, and incidence of serious complications after gastric cancer (Lin et al. 2019). In our study, patients were divided into two 
247 groups based on the CONUT score by calculating a cut-off value, and it was found that the high

248 CONUT score group was more prone to severe postoperative complications and peripancreatic

249 invasion, but was not more likely to develop postoperative pancreatic fistula. The reason for

250 these results may be that the three blood indicators measured under the CONUT score, which

251 reflect the immune and nutritional status of the body, show an increased incidence of serious

252 postoperative complications. However, the development of pancreatic fistula is mostly related to

253 the hardness of the pancreas, whether is accompanied by pancreatitis, surgical technique,

254 anastomosis type, and reconstruction mode, and the effects of immune and nutrition status on

255 pancreatic fistula development seem to be insignificant.

256

257

258

259

260

261

262

263

264

265

266

267

268

269

270

271

272

273

Serum CA199 levels are a classic TMs commonly used in the management of patients with PC (Locker et al. 2006). This study also proved serum CA199 levels to be an independent factor that may predict postoperative survival and prognosis of patients with PDAC. However, serum CA199 levels are elevated not only in the case of PC but also in other cancers and certain inflammatory diseases. Therefore, as a diagnostic tool for PDAC, serum CA199 levels have low sensitivity and specificity (Liu et al. 2019; Zeng et al. 2019). Serum CA199 levels mainly reflects the status of the tumor, whereas the CONUT score reflects the overall status of the patient, including nutritional and immune status. We found that the combination of these two factors (CONUT-CA199 score) may provide more accurate prognostic information for patients with PDAC after surgery than either single factor, as indicated by the present ROC analyses. In addition, the CONUT-CA199 score was shown to be an independent prognostic indicator on multivariate analysis. These results suggest that the combination of serum CA199 levels and the CONUT score is more effective and provides more predictive value than serum CA199 levels or the CONUT score alone in evaluating patients with PDAC after surgery.

However, this study has some limitations. First, the sample size of our study is relatively small. According to the measure of AUCs benchmark(Ceci \& Bjork 2000), the AUC value is lower, this study may be related to poor specificity of CA199 regionalization related cases, inadequate sample size, source, and then through joint COUNT after scoring and CA199 levels, 
274 found the AUC value is increased significantly, close to 0.7, and compared with the single use, 275 obvious advantages, significant difference, therefore, with the enlargement of the sample size 276 and the study population, the COUNT combined CA199 levels is expected to become effective 277 predictor of PDAC survival in patients with postoperative prognosis.

278 In summary, our study is the first to demonstrate that the preoperative CONUT-CA199 279 score is an independent prognostic factor for OS and RFS in patients with PDAC undergoing 280 radical resection. As a novel, economical, and reliable biomarker, the preoperative CONUT281 CA199 score has potential application in the development of individualized treatments and 282 follow-up plans.

283

284

285

286

287

288

289

290

291

292

293

294

295

296

297

\section{Acknowledgment}

This research was funded by the following project funds: Sichuan Science and Technology Plan Project of China (NO. 2018JY0283, 201SZYZF0015), Luzhou Municipal People's Government-Southwest Medical University Science and Technology Strategic Cooperation Applied Basic Research Project (NO. 2018LZXNYD-ZK14), Southwest Medical UniversityLuzhou Chinese Medicine Hospital Basic Project (NO. LZZYYY2018P00039); Southwest Medical University Project (NO. 2018-ZRQN-077)

\section{Competing of interests}

The author states that there is no conflict of interest between them.

\section{Author contributions}

Ankang Wang and Bo Sun wrote the manuscript and performed data analyses; Hao Shi, Zhiwei Huang, Tao He, and Tianxiang Zheng collected the clinical data; Qiu Li, Wenguang Fu, and $\mathrm{Yu}$ Jiang reviewed the manuscript.

\section{References}


Abe T, Nakata K, Kibe S, Mori Y, Miyasaka Y, Ohuchida K, Ohtsuka T, Oda Y, and Nakamura M. 2018. Prognostic Value of Preoperative Nutritional and Immunological Factors in Patients with Pancreatic Ductal Adenocarcinoma. Ann Surg Oncol 25:3996-4003. 10.1245/s10434-018-6761-6

Asama H, Suzuki R, Takagi T, Sugimoto M, Konno N, Watanabe K, Nakamura J, Kikuchi H, Takasumi M, Sato Y, Irie H, Hikichi T, and Ohira H. 2018. Evaluation of inflammation-based markers for predicting the prognosis of unresectable pancreatic ductal adenocarcinoma treated with chemotherapy. Mol Clin Oncol 9:408-414. 10.3892/mco.2018.1696

Balzano G, Dugnani E, Crippa S, Scavini M, Pasquale V, Aleotti F, Liberati D, Gandolfi A, Belfiori G, Reni M, Doglioni C, Ruffo G, Marmorale C, Falconi M, and Piemonti L. 2017. A preoperative score to predict early death after pancreatic cancer resection. Dig Liver Dis 49:1050-1056. 10.1016/j.dld.2017.06.012

Bassi C, Marchegiani G, Dervenis C, Sarr M, Abu Hilal M, Adham M, Allen P, Andersson R, Asbun HJ, Besselink MG, Conlon K, Del Chiaro M, Falconi M, Fernandez-Cruz L, Fernandez-Del Castillo C, Fingerhut A, Friess H, Gouma DJ, Hackert T, Izbicki J, Lillemoe KD, Neoptolemos JP, Olah A, Schulick R, Shrikhande SV, Takada T, Takaori K, Traverso W, Vollmer CR, Wolfgang CL, Yeo CJ, Salvia R, Buehler M, and Surg ISGP. 2017. The 2016 update of the International Study Group (ISGPS) definition and grading of postoperative pancreatic fistula: 11 Years After. Surgery 161:584-591. 10.1016/j.surg.2016.11.014

Berntsson J, Nodin B, Eberhard J, Micke P, and Jirstrom K. 2016. Prognostic impact of tumour-infiltrating B cells and plasma cells in colorectal cancer. Int J Cancer 139:1129-1139. 10.1002/ijc.30138

Borre M, Dam GA, Knudsen AW, and Gronbaek H. 2018. Nutritional status and nutritional risk in patients with neuroendocrine tumors. Scand J Gastroenterol 53:284-292. 10.1080/00365521.2018.1430848

Ceci SJ, and Bjork RA. 2000. Psychological Science in the Public Interest: the case for juried analyses. Psychol Sci 11:177-178. 10.1111/1467-9280.00237

Clavien PA, Barkun J, de Oliveira ML, Vauthey JN, Dindo D, Schulick RD, de Santibanes E, Pekolj J, Slankamenac K, Bassi C, Graf R, Vonlanthen R, Padbury R, Cameron JL, and Makuuchi M. 2009. The Clavien-Dindo classification of surgical complications: five-year experience. Ann Surg 250:187-196. 10.1097/SLA.0b013e3181b13ca2

Duffy MJ, Sturgeon C, Lamerz R, Haglund C, Holubec VL, Klapdor R, Nicolini A, Topolcan O, and Heinemann V. 2010. Tumor markers in pancreatic cancer: a European Group on Tumor Markers (EGTM) status report. Ann Oncol 21:441-447. 10.1093/annonc/mdp332

El-Khayat H, Fouad Y, Mohamed HI, El-Amin H, Kamal EM, Maher M, and Risk A. 2018. Sofosbuvir plus daclatasvir with or without ribavirin in 551 patients with hepatitis C-related cirrhosis, genotype 4. Alimentary Pharmacology \& Therapeutics 47:674-679. 10.1111/apt.14482

Fujioka S, Misawa T, Okamoto T, Gocho T, Futagawa Y, Ishida Y, and Yanaga K. 2007. Preoperative serum 
331

332

333

334

335

336

337

338

339

340

341

342

343

344

345

346

347

348

349

350

351

352

353

354

355

356

357

358

359

360

361

362

363

carcinoembryonic antigen and carbohydrate antigen 19-9 levels for the evaluation of curability and resectability in patients with pancreatic adenocarcinoma. $J$ Hepatobiliary Pancreat Surg 14:539-544. 10.1007/s00534-006-1184-3

Fujiya K, Kawamura T, Omae K, Makuuchi R, Irino T, Tokunaga M, Tanizawa Y, Bando E, and Terashima M. 2018. Impact of Malnutrition After Gastrectomy for Gastric Cancer on Long-Term Survival. Ann Surg Oncol 25:974-983. 10.1245/s10434-018-6342-8

Gilliland TM, Villafane-Ferriol N, Shah KP, Shah RM, Tran Cao HS, Massarweh NN, Silberfein EJ, Choi EA, Hsu C, McElhany AL, Barakat O, Fisher W, and Van Buren G. 2017. Nutritional and Metabolic Derangements in Pancreatic Cancer and Pancreatic Resection. Nutrients 9. 10.3390/nu9030243

Gong Y, Zhang LD, He TY, Ding J, Zhang HY, Chen G, Zhang D, Wu Z, Chen QL, Fan HN, Wang Q, Bie P, and Wang HZ. 2013. Pancreaticoduodenectomy Combined with Vascular Resection and Reconstruction for Patients with Locally Advanced Pancreatic Cancer: A Multicenter, Retrospective Analysis. Plos One 8. ARTN e70340

10.1371/journal.pone.0070340

Gooden MJM, de Bock GH, Leffers N, Daemen T, and Nijman HW. 2011. The prognostic influence of tumourinfiltrating lymphocytes in cancer: a systematic review with meta-analysis. British Journal of Cancer 105:93-103. 10.1038/bjc.2011.189

Haab BB, Huang Y, Balasenthil S, Partyka K, Tang HY, Anderson M, Allen P, Sasson A, Zeh H, Kaul K, Kletter D, Ge SK, Bern M, Kwon R, Blasutig I, Srivastava S, Frazier ML, Sen S, Hollingsworth MA, Rinaudo JA, Killary AM, and Brand RE. 2015. Definitive Characterization of CA 19-9 in Resectable Pancreatic Cancer Using a Reference Set of Serum and Plasma Specimens. Plos One 10. ARTN e0139049

10.1371/journal.pone.0139049

Haghikia A, and Landmesser U. 2018. High-Density Lipoproteins Effects on Vascular Function and Role in the Immune Response. Cardiology Clinics 36:317-+. 10.1016/j.ccl.2017.12.013

Harimoto N, Yoshizumi T, Inokuchi S, Itoh S, Adachi E, Ikeda Y, Uchiyama H, Utsunomiya T, Kajiyama K, Kimura K, Kishihara F, Sugimachi K, Tsujita E, Ninomiya M, Fukuzawa K, Maeda T, Shirabe K, and Maehara Y. 2018. Prognostic Significance of Preoperative Controlling Nutritional Status (CONUT) Score in Patients Undergoing Hepatic Resection for Hepatocellular Carcinoma: A Multi-institutional Study. Ann Surg Oncol 25:3316-3323. 10.1245/s10434-018-6672-6

Ignacio de Ulibarri J, Gonzalez-Madrono A, de Villar NG, Gonzalez P, Gonzalez B, Mancha A, Rodriguez F, and Fernandez G. 2005. CONUT: a tool for controlling nutritional status. First validation in a hospital population. Nutr Hosp 20:38-45.

Iseki Y, Shibutani M, Maeda K, Nagahara H, Ohtani H, Sugano K, Ikeya T, Muguruma K, Tanaka H, Toyokawa T, 
364

365

366

367

368

369

370

371

372

373

374

375

376

377

378

379

380

381

382

383

384

385

386

387

388

389

390

391

392

393

394

395

Sakurai K, and Hirakawa K. 2015. Impact of the Preoperative Controlling Nutritional Status (CONUT) Score on the Survival after Curative Surgery for Colorectal Cancer. Plos One 10:e132488. 10.1371/journal.pone.0132488

Jacobs RJ, Voorneveld PW, Kodach LL, and Hardwick JCH. 2012. Cholesterol metabolism and colorectal cancers. Current Opinion in Pharmacology 12:690-695. 10.1016/j.coph.2012.07.010

Jacobson A. 2006. Type, density, and location of immune cells within human colorectal tumors predict clinical outcome. Science 313:1960-1964.

Kamisawa T, Wood LD, Itoi T, and Takaori K. 2016. Pancreatic cancer. Lancet 388:73-85. 10.1016/S01406736(16)00141-0

Kanda M, Fujii T, Kodera Y, Nagai S, Takeda S, and Nakao A. 2011. Nutritional predictors of postoperative outcome in pancreatic cancer. British Journal of Surgery 98:268-274. 10.1002/bjs.7305

Karamitopoulou E, Zlobec I, Gloor B, Kondi-Pafiti A, Lugli A, and Perren A. 2013. Loss of Raf-1 kinase inhibitor protein (RKIP) is strongly associated with high-grade tumor budding and correlates with an aggressive phenotype in pancreatic ductal adenocarcinoma (PDAC). Journal of Translational Medicine 11. Artn 311

$10.1186 / 1479-5876-11-311$

Kato Y, Yamada S, Suenaga M, Takami H, Niwa Y, Hayashi M, Iwata N, Kanda M, Tanaka C, Nakayama G, Koike M, Fujiwara M, and Kodera Y. 2018. Impact of the Controlling Nutritional Status Score on the Prognosis After Curative Resection of Pancreatic Ductal Adenocarcinoma. Pancreas 47:823-829. 10.1097/MPA.0000000000001105

Liang RF, Li JH, Li M, Yang Y, and Liu YH. 2017. The prognostic role of controlling nutritional status scores in patients with solid tumors. Clin Chim Acta 474:155-158. 10.1016/j.cca.2017.09.021

Lin JX, Lin LZ, Tang YH, Wang JB, Lu J, Chen QY, Cao LL, Lin M, Tu RH, Huang CM, Li P, Zheng CH, and Xie JW. 2019. Which Nutritional Scoring System Is More Suitable for Evaluating the Short- or Long-Term Prognosis of Patients with Gastric Cancer Who Underwent Radical Gastrectomy? J Gastrointest Surg. 10.1007/s11605-019-04360-4

Liu J, Dai Y, Zhou F, Long Z, Li Y, Liu B, Xie D, Tang J, Tan J, Yao K, Zhang Y, Tang Y, and He L. 2016. The prognostic role of preoperative serum albumin/globulin ratio in patients with bladder urothelial carcinoma undergoing radical cystectomy. Urol Oncol 34:484 e481-484 e488. 10.1016/j.urolonc.2016.05.024

Liu XC, Zhang DY, Lin EZ, Chen YM, Li W, Chen YB, Sun XW, and Zhou ZW. 2018. Preoperative controlling nutritional status (CONUT) score as a predictor of long-term outcome after curative resection followed by adjuvant chemotherapy in stage II-III gastric Cancer. Bmc Cancer 18. ARTN 699

$10.1186 / \mathrm{s} 12885-018-4616-\mathrm{y}$

Peer) reviewing PDF | (2019:09:41662:1:1:CHECK 19 Jan 2020) 
396

Liu YW, Feng W, Liu WY, Kong XY, Li L, He JB, Wang DW, Zhang MT, Zhou G, Xu W, Chen W, Gong AH, and $\mathrm{Xu}$ M. 2019. Circulating lncRNA ABHD11-AS1 serves as a biomarker for early pancreatic cancer diagnosis. Journal of Cancer 10:3746-3756. 10.7150/jca.32052

Locker GY, Stanley H, Jules H, Jessup JM, Nancy K, Macdonald JS, Somerfield MR, Hayes DF, and Bast RC. 2006. ASCO 2006 update of recommendations for the use of tumor markers in gastrointestinal cancer. Journal of Clinical Oncology 24:5313-5327.

Mantzorou M, Koutelidakis A, Theocharis S, and Giaginis C. 2017. Clinical Value of Nutritional Status in Cancer: What is its Impact and how it Affects Disease Progression and Prognosis? Nutrition and Cancer-an International Journal 69:1151-1176. 10.1080/01635581.2017.1367947

Mohri Y, Inoue Y, Tanaka K, Hiro J, Uchida K, and Kusunoki M. 2013. Prognostic Nutritional Index Predicts Postoperative Outcome in Colorectal Cancer. World Journal of Surgery 37:2688-2692. 10.1007/s00268013-2156-9

Ni XC, Xu J, Yi Y, Fu YP, Cai XY, Liu G, Huang JL, Gan W, and Qiu SJ. 2019. Inflammation-nutrition score predicts prognosis of patients with resectable hepatocellular carcinoma. Int J Clin Oncol 24:825-835. 10.1007/s10147-019-01402-4

O'Brien DP, Sandanayake NS, Jenkinson C, Gentry-Maharaj A, Apostolidou S, Fourkala EO, Camuzeaux S, Blyuss O, Gunu R, Dawnay A, Zaikin A, Smith RC, Jacobs IJ, Menon U, Costello E, Pereira SP, and Timms JF. 2015. Serum CA19-9 Is Significantly Upregulated up to 2 Years before Diagnosis with Pancreatic Cancer: Implications for Early Disease Detection. Clinical Cancer Research 21:622-631. 10.1158/1078-0432.Ccr14-0365

Pancreatic Cancer Committee of Chinese Anti-Cancer A. 2018. [Comprehensive guidelines for the diagnosis and treatment of pancreatic cancer (2018 version)]. Zhonghua Wai Ke Za Zhi 56:481-494. 10.3760/cma.j.issn.0529-5815.2018.07.001

Pinato DJ, North BV, and Sharma R. 2012. A novel, externally validated inflammation-based prognostic algorithm in hepatocellular carcinoma: the prognostic nutritional index (PNI). $\mathrm{Br} J$ Cancer 106:1439-1445. $10.1038 /$ bjc. 2012.92

Rahib L, Smith BD, Aizenberg R, Rosenzweig AB, Fleshman JM, and Matrisian LM. 2014. Projecting cancer incidence and deaths to 2030: the unexpected burden of thyroid, liver, and pancreas cancers in the United States. Cancer Res 74:2913-2921. 10.1158/0008-5472.CAN-14-0155

Satake K, Kanazawa G, Kho I, Chung YS, and Umeyama K. 1985. A clinical evaluation of carbohydrate antigen 199 and carcinoembryonic antigen in patients with pancreatic carcinoma. J Surg Oncol 29:15-21.

Shoji F, Haratake N, Akamine T, Takamori S, Katsura M, Takada K, Toyokawa G, Okamoto T, and Maehara Y. 2017. The Preoperative Controlling Nutritional Status Score Predicts Survival After Curative Surgery in 
429

430

431

432

433

434

435

436

437

438

439

440

441

442

443

444

445

446

447

448

449

450

451

452

453

454

455

456

457

458

459

460

Patients with Pathological Stage I Non-small Cell Lung Cancer. Anticancer Res 37:741-747. 10.21873/anticanres.11372

Siegel RL, Miller KD, and Jemal A. 2019. Cancer statistics, 2019. CA Cancer J Clin 69:7-34. 10.3322/caac.21551

Staal B, Liu Y, Barnett D, Hsueh P, He Z, Gao C, Partyka K, Hurd MW, Singhi AD, Drake RR, Huang Y, Maitra A, Brand RE, and Haab BB. 2019. The sTRA Plasma Biomarker: Blinded Validation of Improved Accuracy Over CA19-9 in Pancreatic Cancer Diagnosis. Clin Cancer Res 25:2745-2754. 10.1158/1078-0432.CCR18-3310

Sun KY, Xu JB, Chen SL, Yuan YJ, Wu H, Peng JJ, Chen CQ, Guo P, Hao YT, and He YL. 2015. Novel immunological and nutritional-based prognostic index for gastric cancer. World Journal of Gastroenterology 21:5961-5971. 10.3748/wjg.v21.119.5961

Takagi K, Yagi T, Umeda Y, Shinoura S, Yoshida R, Nobuoka D, Kuise T, Araki H, and Fujiwara T. 2017. Preoperative Controlling Nutritional Status (CONUT) Score for Assessment of Prognosis Following Hepatectomy for Hepatocellular Carcinoma. World J Surg 41:2353-2360. 10.1007/s00268-017-3985-8

Tang YC, Xu XJ, Guo SX, Zhang CB, Tang Y, Tian Y, Ni B, Lu BF, and Wang HZ. 2014. An Increased Abundance of Tumor-Infiltrating Regulatory T Cells Is Correlated with the Progression and Prognosis of Pancreatic Ductal Adenocarcinoma. Plos One 9. ARTN e91551

10.1371/journal.pone.0091551

Tokunaga R, Sakamoto Y, Nakagawa S, Ohuchi M, Izumi D, Kosumi K, Taki K, Higashi T, Miyamoto Y, Yoshida N, Oki E, Watanabe M, and Baba H. 2017. CONUT: a novel independent predictive score for colorectal cancer patients undergoing potentially curative resection. Int J Colorectal Dis 32:99-106. 10.1007/s00384016-2668-5

van Dijk M, and Pot GK. 2016. The effects of nutritional interventions on recurrence in survivors of colorectal adenomas and cancer: a systematic review of randomised controlled trials. Eur J Clin Nutr 70:566-573. 10.1038/ejcn.2015.210

van Roessel S, Kasumova GG, Verheij J, Najarian RM, Maggino L, de Pastena M, Malleo G, Marchegiani G, Salvia R, Ng SC, de Geus SW, Lof S, Giovinazzo F, van Dam JL, Kent TS, Busch OR, van Eijck CH, Koerkamp BG, Abu Hilal M, Bassi C, Tseng JF, and Besselink MG. 2018. International Validation of the Eighth Edition of the American Joint Committee on Cancer (AJCC) TNM Staging System in Patients With Resected Pancreatic Cancer. Jama Surgery 153. ARTN e183617

10.1001/jamasurg.2018.3617

Winter JM, Yeo CJ, and Brody JR. 2013. Diagnostic, prognostic, and predictive biomarkers in pancreatic cancer. $J$ Surg Oncol 107:15-22. 10.1002/jso.23192 
461

Wu H, Guo JC, Yang SH, Tien YW, and Kuo SH. 2019. Postoperative Imaging and Tumor Marker Surveillance in Resected Pancreatic Cancer. J Clin Med 8. 10.3390/jcm8081115

Xiao H, Zhou H, Zhang P, Xiao H, Liu K, Chen X, Quan H, Yin B, Li R, Huang G, Yin X, and Ouyang Y. 2019. Association among the prognostic nutritional index, completion of adjuvant chemotherapy, and cancerspecific survival after curative resection of stage II/III gastric cancer. Eur J Clin Nutr. 10.1038/s41430-019$0502-1$

Yamamoto M, Saito H, Uejima C, Tanio A, Tada Y, Matsunaga T, Sakamoto T, Honjo S, Ashida K, and Fujiwara Y. 2019. Prognostic Value of Combined Tumor Marker and Controlling Nutritional Status (CONUT) Score in Colorectal Cancer Patients. Yonago Acta Medica 62:124-130. DOI 10.33160/yam.2019.03.017

Yang C, Wei C, Wang S, Han S, Shi D, Zhang C, Lin X, Dou R, and Xiong B. 2019a. Combined Features Based on Preoperative Controlling Nutritional Status Score and Circulating Tumour Cell Status Predict Prognosis for Colorectal Cancer Patients Treated with Curative Resection. Int J Biol Sci 15:1325-1335. 10.7150/ijbs.33671

Yang CG, Wei C, Wang SY, Han S, Shi DD, Zhang CX, Lin XB, Dou RZ, and Xiong B. 2019b. Combined Features Based on Preoperative Controlling Nutritional Status Score and Circulating Tumour Cell Status Predict Prognosis for Colorectal Cancer Patients Treated with Curative Resection. International Journal of Biological Sciences 15:1325-1335. 10.7150/ijbs.33671

Zeng P, Li H, Chen Y, Pei H, and Zhang L. 2019. Serum CA199 levels are significantly increased in patients suffering from liver, lung, and other diseases. Prog Mol Biol Transl Sci 162:253-264. 10.1016/bs.pmbts.2018.12.010 


\section{Table $\mathbf{1}$ (on next page)}

Scoring system for the controlling nutritional status ( CONUT )

$*$ CONUT score $=$ Serum albumin score + total lym phocyte score + total cholesterol score 
1

2 Table1 Scoring system for the controlling nutritional status (CONUT)

\begin{tabular}{ccccc}
\hline Degree of undernutrition & CONUT score & $\begin{array}{c}\text { Serum albumin } \\
(\mathrm{g} / \mathrm{dl})\end{array}$ & $\begin{array}{c}\text { Total lymphocyte } \\
\left(/ \mathrm{mm}^{3}\right)\end{array}$ & $\begin{array}{c}\text { Total cholesterol } \\
(\mathrm{mg} / \mathrm{dl})\end{array}$ \\
Normal & $0-1$ & $\geq 3.50(0)$ & $\geq 1600(0)$ & $\geq 180(0)$ \\
Mild & $2-4$ & $3.00-3.49(2)$ & $1200-1599(1)$ & $140-179(1)$ \\
Moderate & $5-8$ & $2.50-2.99(4)$ & $800-1199(2)$ & $100-139(2)$ \\
Severe & $9-12$ & $<2.50(6)$ & $<800(3)$ & $<100(3)$ \\
\hline
\end{tabular}

$3 *$ CONUT score $=$ Serum albumin score + total lymphocyte score + total cholesterol score 


\section{Table 2 (on next page)}

Relationships between CONUTscore and clinicopathological characteristics of 294 PDAC patients

Notes: *indicates P<0.05. PDAC, Pancreatic ductal adenocarcinoma; BMI, body mass index; p TNM , Pathologic tumour-node-metastasis; CONUT, controlling nutritional status; The cut off value of CONUT score is 3, according to the ROC analyses; 
1 Table 2 Relationships between CONUT score and clinicopathological characteristics of 294 PDAC 2 patients

\begin{tabular}{|c|c|c|c|c|}
\hline Variable & $\begin{array}{c}\text { CONUT }^{\text {low }} \\
\qquad(n=194)\end{array}$ & $\begin{array}{l}\text { CONUT } \\
(n=100)\end{array}$ & $\chi^{2}$ value & $P$ value \\
\hline Gender & & & 0.401 & 0.526 \\
\hline Male & $105(54 \%)$ & $58(58 \%)$ & & \\
\hline Female & $89(46 \%)$ & $42(42 \%)$ & & \\
\hline Age (years) & & & 3.205 & 0.073 \\
\hline$<52$ & $87(45 \%)$ & $34(34 \%)$ & & \\
\hline$\geq 52$ & $107(55 \%)$ & $66(66 \%)$ & & \\
\hline BMI (kg/m2) & & & 3.455 & 0.178 \\
\hline$<18.5$ & $36(19 \%)$ & $25(25 \%)$ & & \\
\hline$\geq 18.5,<25.0$ & $128(66 \%)$ & $61(61 \%)$ & & \\
\hline$\geq 25.0$ & $30(15 \%)$ & $14(14 \%)$ & & \\
\hline Tumour location & & & 3.036 & 0.219 \\
\hline Pancreatic head & $145(75 \%)$ & $69(69 \%)$ & & \\
\hline Pancreatic body and tail & $41(21 \%)$ & $22(22 \%)$ & & \\
\hline Dispersed & $8(4 \%)$ & $9(9 \%)$ & & \\
\hline Tumor size $(\mathrm{cm})$ & & & 1.925 & 0.165 \\
\hline$<3.1$ & $134(69 \%)$ & $61(61 \%)$ & & \\
\hline$\geq 3.1$ & $60(31 \%)$ & $39(39 \%)$ & & \\
\hline Histopathological type & & & 2.546 & 0.111 \\
\hline Poorly differentiated & $80(41 \%)$ & $51(51 \%)$ & & \\
\hline medium-high differentiation & $114(59 \%)$ & $49(49 \%)$ & & \\
\hline Peripancreatic infiltration & & & 4.447 & $0.035 *$ \\
\hline Positive & $144(74 \%)$ & $85(85 \%)$ & & \\
\hline Negative & $50(26 \%)$ & $15(15 \%)$ & & \\
\hline Lymph node metastasis & & & 0.866 & 0.352 \\
\hline Positive & $84(43 \%)$ & $49(49 \%)$ & & \\
\hline Negative & $110(57 \%)$ & $51(51 \%)$ & & \\
\hline Lymphatic vessel invasion & & & 0.365 & 0.546 \\
\hline Positive & $146(75 \%)$ & $72(72 \%)$ & & \\
\hline Negative & $48(25 \%)$ & $28(28 \%)$ & & \\
\hline Invasion of portal vein system & & & 2.356 & 0.125 \\
\hline Positive & $53(27 \%)$ & $36(36 \%)$ & & \\
\hline Negative & $141(73 \%)$ & $64(64 \%)$ & & \\
\hline Superior mesenteric artery invasion & & & 1.522 & 0.217 \\
\hline Positive & $58(30 \%)$ & $37(37 \%)$ & & \\
\hline Negative & $136(70 \%)$ & $63(63 \%)$ & & \\
\hline Nerve plexus invasion & & & 1.081 & 0.299 \\
\hline
\end{tabular}




\begin{tabular}{|c|c|c|c|c|}
\hline Positive & $120(62 \%)$ & $68(68 \%)$ & & \\
\hline Negative & $74(38 \%)$ & $32(32 \%)$ & & \\
\hline pTNM stage & & & 3.642 & 0.056 \\
\hline I-II & $136(70 \%)$ & $59(59 \%)$ & & \\
\hline III & $58(30 \%)$ & $41(41 \%)$ & & \\
\hline Clavien-Dindo classification & & & 24.342 & $<0.001 *$ \\
\hline$<$ IIIIa & $170(88 \%)$ & $63(63 \%)$ & & \\
\hline$\geq \mathrm{IIIIa}$ & $24(12 \%)$ & $37(37 \%)$ & & \\
\hline Pancreatic fistula & & & 0.269 & 0.604 \\
\hline Presence & $34(18 \%)$ & $20(20 \%)$ & & \\
\hline Absence & $160(82 \%)$ & $80(80 \%)$ & & \\
\hline
\end{tabular}

3 Notes: *indicates $\mathrm{P}<0.05$. PDAC, Pancreatic ductal adenocarcinoma; BMI, body mass index; 4 pTNM, Pathologic tumour-node-metastasis; CONUT, controlling nutritional status; The cut off 5 value of CONUT score is 3 , according to the ROC analyses; 


\section{Table 3(on next page)}

Univariate analyses offactors associated with overall survival and recurrence-free survival of PDACpatients

Notes: *indicates $\mathrm{P}<0.05$. PDAC, Pancreatic ductal adenocarcinoma; HR, hazard ratio; $\mathrm{Cl}$, confidence interval; OS, overall survival; RFS, recurrence-free survival; BMI, body mass index; $\mathrm{p}$ TNM, Pathologic tumour-node-metastasis; CEA, carcinoembryonic antigen; CA199; carbohydrate antigen 199; PNI, prognostic nutritional index; CONUT, controlling nutritional status; 
1 Table 3 Univariate analyses of factors associated with overall survival and recurrence-free 2 survival of PDAC patients

\begin{tabular}{|c|c|c|c|c|c|c|}
\hline \multirow{2}{*}{ Variable } & \multicolumn{3}{|c|}{ OS } & \multicolumn{3}{|c|}{ RFS } \\
\hline & HR & $95 \% \mathrm{CI}$ & $P$ value & HR & $95 \%$ CI & $P$ value \\
\hline Gender (Male vs. Female) & 0.78 & $0.57-1.08$ & 0.137 & 0.85 & $0.63-1.16$ & 0.311 \\
\hline Age (<52 vs. $\geq 52$ years $)$ & 1.51 & $1.08-2.11$ & $0.015^{*}$ & 1.49 & $1.09-2.03$ & $0.013^{*}$ \\
\hline Preoperative CEA (<6.4 vs. $\geq 6.4$ ng/ml) & 1.41 & $0.95-2.11$ & 0.092 & 1.29 & $0.87-1.91$ & 0.200 \\
\hline Preoperative CA199 (<36.6 vs. $\geq 36.6 \mathrm{ng} / \mathrm{ml}$ ) & 3.03 & $2.15-4.27$ & $<0.001^{*}$ & 2.22 & $1.63-3.04$ & $<0.001 *$ \\
\hline BMI ( $<18.5$ vs. $\geq 18.5,<25.0$ vs. $\geq 25.0 \mathrm{~kg} / \mathrm{m} 2)$ & 1.10 & $0.85-1.44$ & 0.471 & 1.04 & $0.81-1.33$ & 0.789 \\
\hline Tumour size (<3.1 vs. $\geq 3.1 \mathrm{~cm})$ & 1.49 & $1.07-2.07$ & $0.018^{*}$ & 1.58 & $1.16-2.16$ & $0.004 *$ \\
\hline Tumour Location (Head vs. Body and tail vs. Dispersed) & 0.89 & $0.68-1.17$ & 0.402 & 0.83 & $0.63-1.08$ & 0.157 \\
\hline Histopathological type (Poor vs. medium-high) & 0.54 & $0.38-0.75$ & $<0.001^{*}$ & 0.55 & $0.40-0.80$ & $<0.001^{*}$ \\
\hline Peripancreatic infiltration (Positive vs. Negative) & 0.34 & $0.21-0.53$ & $<0.001^{*}$ & 0.40 & $0.26-0.59$ & $<0.001^{*}$ \\
\hline Lymph node metastasis (Positive vs. Negative) & 0.28 & $0.20-0.41$ & $<0.001^{*}$ & 0.31 & $0.22-0.43$ & $<0.001^{*}$ \\
\hline Lymphatic vessel invasion (Positive vs. Negative) & 0.92 & $0.62-1.35$ & 0.657 & 0.93 & $0.65-1.33$ & 0.686 \\
\hline Superior mesenteric artery invasion (Positive vs. Negative) & 0.45 & $0.32-0.63$ & $<0.001^{*}$ & 0.50 & $0.36-0.69$ & $<0.001 *$ \\
\hline Invasion of portal vein system (Positive vs. Negative) & 0.55 & $0.39-0.78$ & $0.001 *$ & 0.54 & $0.39-0.76$ & $<0.001^{*}$ \\
\hline Nerve plexus invasion (Positive vs. Negative) & 0.67 & $0.48-0.95$ & $0.023^{*}$ & 0.66 & $0.48-0.90$ & $0.010^{*}$ \\
\hline pTNM stage (I-II vs. III) & 2.86 & $2.05-4.00$ & $<0.001^{*}$ & 2.44 & $1.78-3.35$ & $<0.001 *$ \\
\hline Clavien-Dindo classification ( $<$ IIIa vs. $\geq$ IIIa) & 1.54 & $1.06-2.24$ & 0.123 & 1.42 & $0.99-2.04$ & 0.059 \\
\hline Pancreatic fistula (Presence vs. Absence) & 0.92 & $0.62-1.38$ & 0.700 & 0.94 & $0.64-1.38$ & 0.749 \\
\hline Preoperative $\mathrm{PNI}(<46.1$ vs. $\geq 46.1)$ & 0.51 & $0.37-0.71$ & $<0.001^{*}$ & 0.58 & $0.43-0.79$ & $0.001 *$ \\
\hline Preoperative COUNT score (Low vs. High) & 3.50 & $2.52-4.87$ & $<0.001^{*}$ & 2.68 & $1.95-3.69$ & $<0.001^{*}$ \\
\hline Preoperative CONUT-CA199 score (1 vs. 2 vs. 3 vs. 4 ) & 2.10 & $1.81-2.44$ & $<0.001 *$ & 1.78 & $1.54-2.05$ & $<0.001^{*}$ \\
\hline
\end{tabular}

3 Notes: *indicates $\mathrm{P}<0.05$. PDAC, Pancreatic ductal adenocarcinoma; HR, hazard ratio; CI, 4 confidence interval; OS, overall survival; RFS, recurrence-free survival; BMI, body mass index; 5 pTNM, Pathologic tumour-node-metastasis; CEA, carcinoembryonic antigen; CA199; 6 carbohydrate antigen 199; PNI, prognostic nutritional index; CONUT, controlling nutritional 7 status; 


\section{Table 4(on next page)}

Multivariate analyses offactors associated with overall survival and recurrence-free survival of PDACpatients

Notes: *indicates $\mathrm{P}<0.05$. PDAC, Pancreatic ductal adenocarcinoma ; HR, hazard ratio; $\mathrm{Cl}$, confidence interval; OS, overall survival; DFS, disease- freesurvival; p TNM, Pathologic tumour-node-metastasis; CEA, carcinoembryonic antigen; CA199; carbohydrate antigen 199; PNI, prognostic nutritional index; CONUT, controlling nutritional status; 
1 Table 4 Multivariate analyses of factors associated with overall survival and recurrence-free 2 survival of PDAC patients

\begin{tabular}{|c|c|c|c|c|c|c|}
\hline \multirow{2}{*}{ Variable } & \multicolumn{3}{|c|}{ OS } & \multicolumn{3}{|c|}{ DFS } \\
\hline & HR & $95 \%$ CI & $P$ value & HR & $95 \%$ CI & $P$ value \\
\hline \multicolumn{7}{|l|}{ Model 1} \\
\hline Age ( $<52$ vs. $\geq 52$ years $)$ & 0.98 & $0.68-1.42$ & 0.933 & 0.89 & $0.61-1.30$ & 0.546 \\
\hline Tumour size (<3.1 vs. $\geq 3.1 \mathrm{~cm})$ & 1.11 & $0.76-1.62$ & 0.608 & 1.09 & $0.74-1.60$ & 0.657 \\
\hline Histopathological type (Poor vs. medium-high) & 0.94 & $0.65-1.36$ & 0.735 & 1.11 & $0.76-1.61$ & 0.590 \\
\hline Peripancreatic infiltration (Positive vs. Negative) & 0.60 & $0.38-0.94$ & $0.027^{*}$ & 0.60 & $0.40-0.91$ & $0.017^{*}$ \\
\hline Lymph node metastasis (Positive vs. Negative) & 0.33 & $0.23-0.49$ & $<0.001 *$ & 0.37 & $0.26-0.53$ & $<0.001^{*}$ \\
\hline Superior mesenteric artery invasion (Positive vs. Negative) & 1.07 & $0.62-1.86$ & 0.800 & 1.11 & $0.64-1.93$ & 0.707 \\
\hline Invasion of portal vein system (Positive vs. Negative) & 0.85 & $0.58-1.25$ & 0.411 & 0.77 & $0.52-1.14$ & 0.193 \\
\hline Nerve plexus invasion (Positive vs. Negative) & 0.85 & $0.59-1.24$ & 0.408 & 0.98 & $0.68-1.42$ & 0.914 \\
\hline pTNM stage (I-II vs. III) & 1.87 & $1.30-2.70$ & $0.001 *$ & 1.63 & $1.16-2.29$ & $0.005^{*}$ \\
\hline Preoperative $\mathrm{PNI}(<46.1$ vs. $\geq 46.1)$ & 0.89 & $0.62-1.29$ & 0.548 & 0.81 & $0.55-1.20$ & 0.289 \\
\hline Preoperative COUNT score (Low vs. High) & 4.00 & $2.82-5.67$ & $<0.001 *$ & 2.93 & $2.10-4.10$ & $<0.001^{*}$ \\
\hline Preoperative CA199 (<36.6 vs. $\geq 36.6 \mathrm{ng} / \mathrm{ml})$ & 2.23 & $1.57-3.17$ & $<0.001 *$ & 1.66 & $1.20-2.29$ & $0.002 *$ \\
\hline \multicolumn{7}{|l|}{ Model 2} \\
\hline Age $(<52$ vs. $\geq 52$ years $)$ & 0.99 & $0.69-1.43$ & 0.951 & 0.96 & $0.68-1.37$ & 0.832 \\
\hline Tumour size $(<3.1$ vs. $\geq 3.1 \mathrm{~cm})$ & 1.11 & $0.76-1.62$ & 0.593 & 1.20 & $0.84-1.71$ & 0.309 \\
\hline Histopathological type (Poor vs. medium-high) & 0.92 & $0.64-1.33$ & 0.661 & 0.94 & $0.67-1.33$ & 0.723 \\
\hline Peripancreatic infiltration (Positive vs. Negative) & 0.59 & $0.37-0.94$ & $0.026^{*}$ & 0.60 & $0.40-0.91$ & $0.017^{*}$ \\
\hline Lymph node metastasis (Positive vs. Negative) & 0.33 & $0.22-0.49$ & $<0.001 *$ & 0.37 & $0.26-0.53$ & $<0.001^{*}$ \\
\hline Superior mesenteric artery invasion (Positive vs. Negative) & 1.06 & $0.61-1.83$ & 0.839 & 1.12 & $0.65-1.92$ & 0.689 \\
\hline Invasion of portal vein system (Positive vs. Negative) & 0.86 & $0.59-1.26$ & 0.434 & 0.78 & $0.54-1.11$ & 0.164 \\
\hline Nerve plexus invasion (Positive vs. Negative) & 0.85 & $0.58-1.24$ & 0.412 & 0.83 & $0.59-1.18$ & 0.303 \\
\hline pTNM stage (I-II vs. III) & 1.91 & $1.33-2.73$ & $<0.001 *$ & 1.62 & $1.16-2.27$ & $0.005^{*}$ \\
\hline Preoperative $\mathrm{PNI}(<46.1$ vs. $\geq 46.1)$ & 0.90 & $0.62-1.30$ & 0.576 & 0.94 & $0.66-1.32$ & 0.701 \\
\hline Preoperative CONUT-CA199 score (1 vs. 2 vs. 3 vs.4) & 2.04 & $1.74-2.40$ & $<0.001^{*}$ & 1.70 & $1.46-1.98$ & $<0.001^{*}$ \\
\hline
\end{tabular}

3 Notes: *indicates $\mathrm{P}<0.05$. PDAC, Pancreatic ductal adenocarcinoma; HR, hazard ratio; CI, 4 confidence interval; OS, overall survival; DFS, disease- freesurvival; pTNM, Pathologic tumour5 node-metastasis; CEA, carcinoembryonic antigen; CA199; carbohydrate antigen 199; PNI, 6 prognostic nutritional index; CONUT, controlling nutritional status; 
Figure 1

Fig. 1. Overall survival curves for pancreatic ductal adenocarcinoma patients according to CONUT score (A) and serum CA199 level (B).

CONUT, Controlling Nutritional Status; CA199; carbohydrate antigen 199.
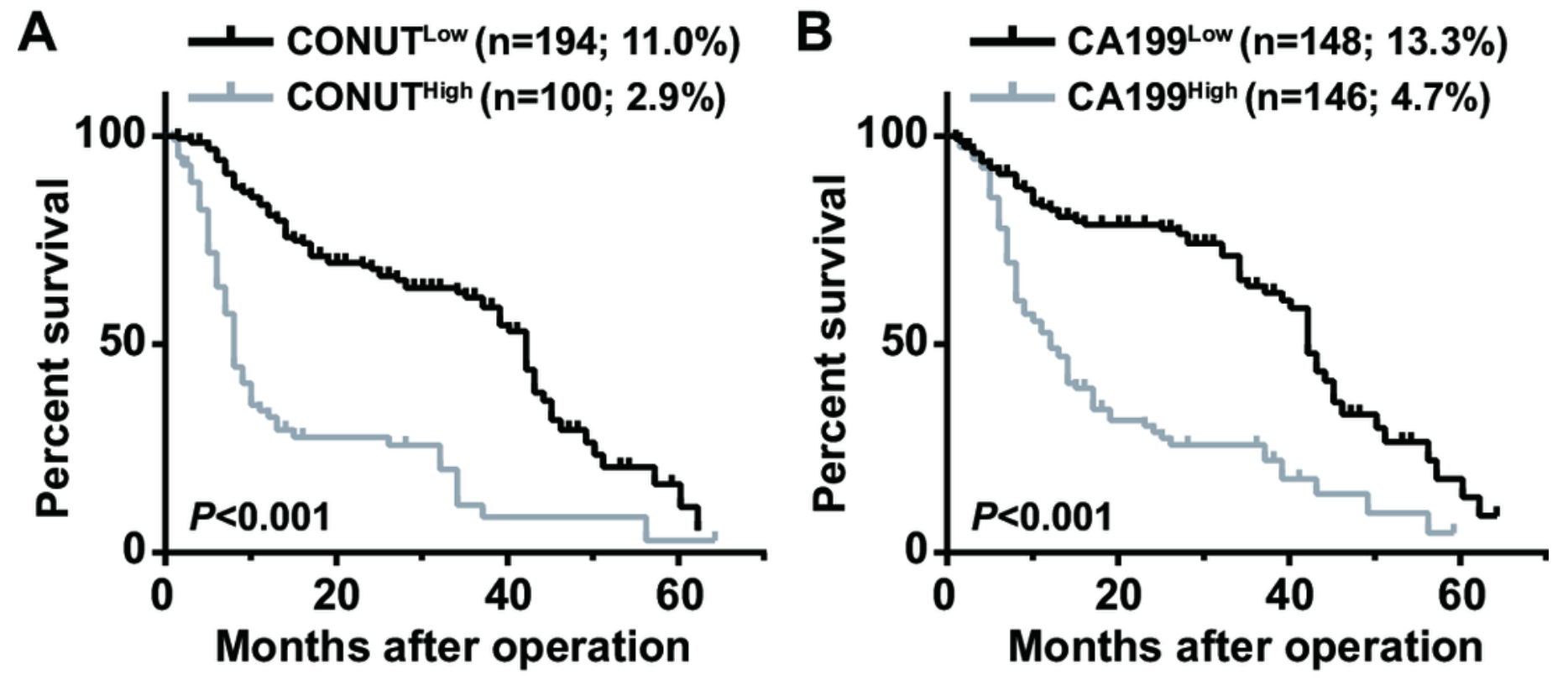
Figure 2

Fig. 2. Overall survival curves (A) and recurrence-free survival curves (B) for pancreatic ductal adenocarcinoma patients according to the combination of CONUT score and serum CA199 level.

CONUT, Controlling Nutritional Status; CA199; carbohydrate antigen 199.
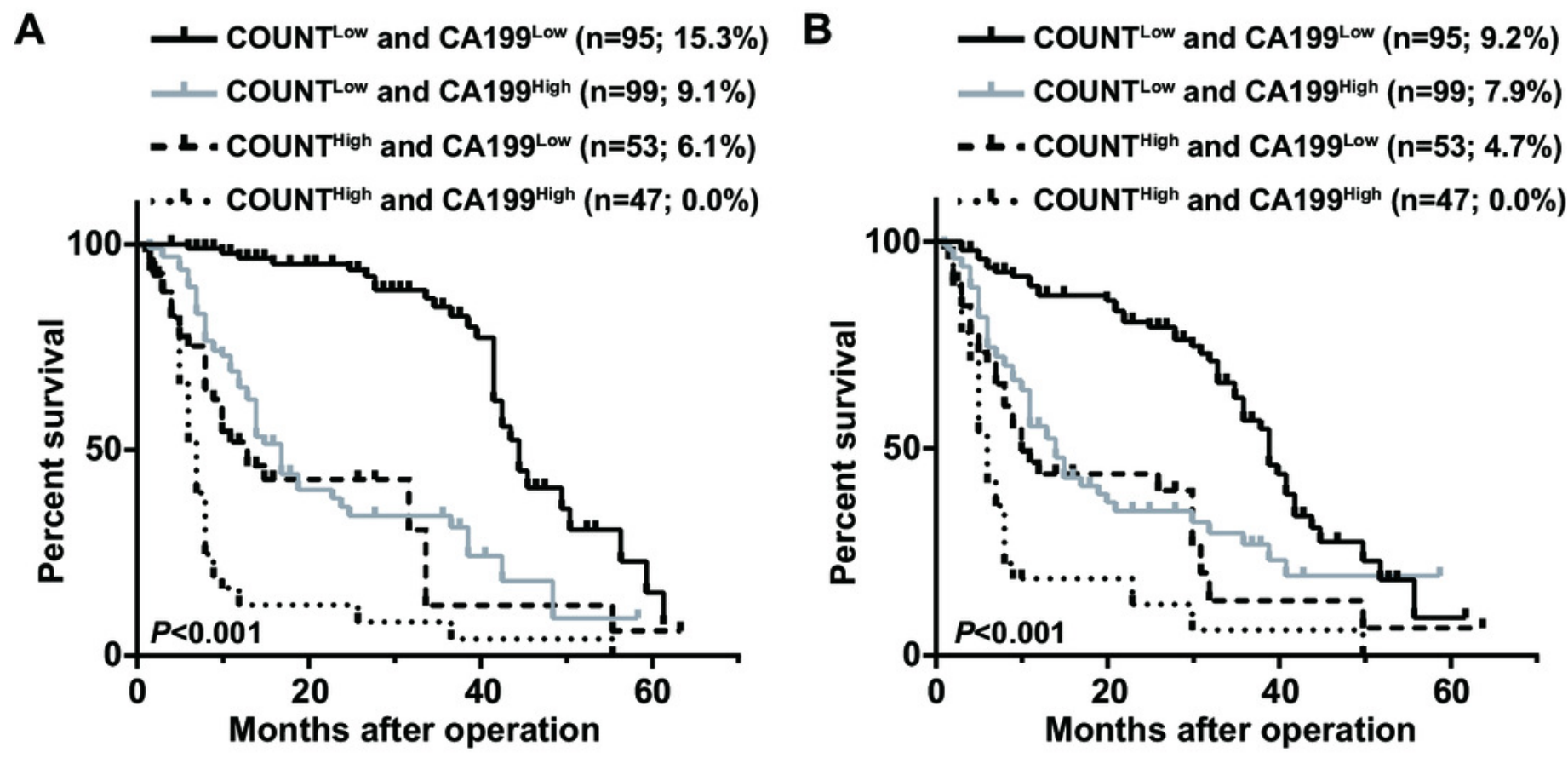
Figure 3

Fig. 3. Time-dependent ROCcurves of preoperative CONUT score, serum CA199 level, and CONUT-CA199 scorefor the prediction of pancreatic ductal adenocarcinoma patients' outcomes.

A. Overall survival . B. Recurrence-free survival. CONUT, controlling nutritional status; CA199; carbohydrate antigen 199; ROC, receiver operating characteristic; AUC, area under curve; $\mathrm{Cl}$, confidence interval.
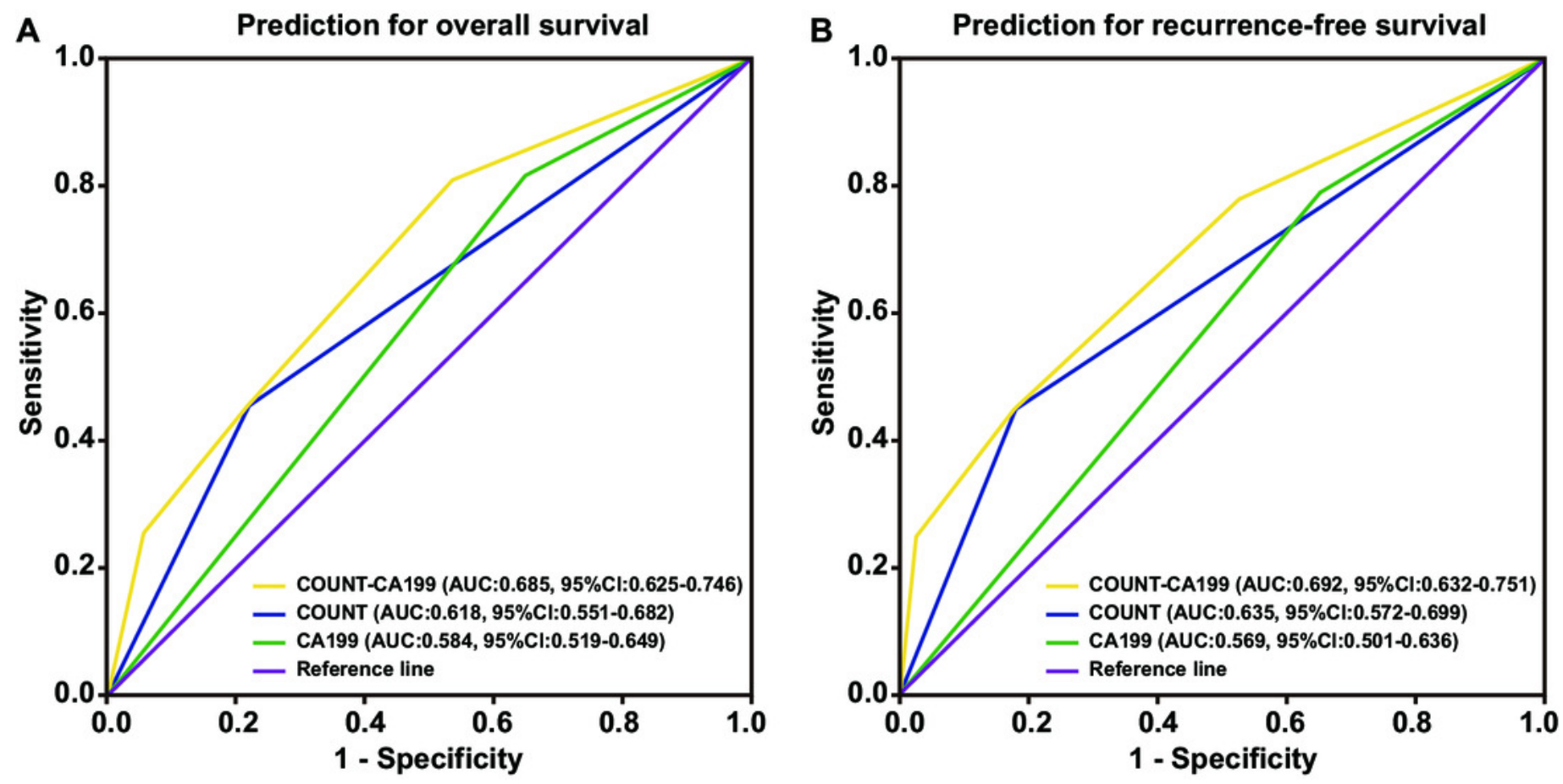